\title{
Éditorial
}

Rejoindrons-nous

le Benelux en matière

de suicide assisté ?

Shall we go along with

Benelux on assisted suicide?

\author{
Jean-Marc GUILÉ \\ Rédacteur en chef
}

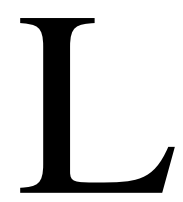

a question de l'accompagnement de la fin de vie vient de resurgir, avec le mot d'ordre de certains groupes de copier les Pays-Bas.

Les trois pays européens formant le Benelux ont légalisé l'euthanasie active et le suicide assisté ${ }^{1}$, la Suisse ayant dépénalisé le suicide assisté, mais pas l'euthanasie (pour une revue, voir Grosse et Grosse, 2014 ; Van der Heide, 2013). Les Pays-Bas ont développé une pratique antérieure à la législation de 2002 portée par les médecins (Royal Dutch Medical Association, KNMG) sur la base d'un changement de paradigme, l'objectif médical n'étant pas de retarder la mort mais d'abréger les souffrances. Outre la capacité de discernement, deux cri-

1. La législation est appliquée aux Pays-Bas, Belgique et Luxembourg depuis 2002, 2002 et 2009 respectivement. tères sont requis : un critère objectif, l'incurabilité de la maladie, indistinctement somatique ou mentale, et un critère subjectif, le caractère insupportable des souffrances psychiques ou somatiques.

Plusieurs auteurs sont préoccupés de voir la législation évoluer sur une pente glissante (slippery slope) (voir Van der Heide, 2013, pour une revue critique). Deux questions concernent plus particulièrement notre champ.

\section{La question de l'âge}

La législation des Pays-Bas (Termination of Life on Request and Assisted Suicide Act 2002) donne accès au suicide assisté aux mineurs de 12 à 15 ans lorsque les titulaires de l'autorité parentale y consentent, et aux mineurs de 16 à 17 ans sans obligation de consentement parental, les parents ayant un avis consultatif (Netherlands Ministry 
of Foreign Affairs, 2010). En février 2014, la Belgique a étendu aux mineurs, sans limite d'âge, le bénéfice de la loi belge de 2002 sur l'euthanasie. Cette modification législative suit la position d'auteurs du groupe sur les soins en fin de vie, affilié aux centres de Bruxelles et Gand de l'Université Hollandaise Vrije, pour lesquels toute limite d'âge fixée a priori est arbitraire, les capacités de discernement devant être évaluées au cas par cas (Chambaere et al., 2014). D'autres auteurs, refusent de placer l'enfant devant un tel choix ou reconnaissent à l'enfance un état d'innocence, et récusent cette compétence de discernement aux enfants, arguant de faits cliniques (Siegel et al., 2014) ou neurobiologiques (Mendelson et Haywood, 2014). Dans l'état actuel de la législation belge, la demande du mineur mène à une évaluation du discernement par un pédopsychiatre ou un psychologue. La décision est soumise à l'accord parental.

\section{La question psychique}

Elle se pose pour le critère subjectif de la souffrance ressentie, comme pour la nature de la maladie incurable, cette dernière pouvant être aussi bien somatique que psychiatrique. La loi hollandaise donne accès au suicide assisté aux patients porteurs d'une affection psychiatrique chronique (Netherlands Ministry of Foreign Affairs, 2010). En 2011, 3695 cas d'euthanasie ou suicide assisté ont été rapportés ; 13 cas concernaient des troubles psychiatriques et 49 un syndrome démentiel
(Grosse et Grosse, 2014). Si, psychologue ou psychiatre, nous sommes appelés à intervenir dans le futur, non plus dans le contexte de la dépénalisation ancienne du suicide, mais sur un « droit au suicide», revendiqué comme tel par certains groupes et légalisé ensuite en France, nul doute qu'il ne s'agira pas uniquement d'apprécier le discernement du demandeur, mais tout autant les phénomènes intersubjectifs et la subjectivité de l'examinateur.

Cet état de fait, à nos portes, ne peut manquer de nous émouvoir, de questionner nos repères cliniques et de nous presser d'y réfléchir.

\section{RÉFÉRENCES BIBLIOGRAPHIQUES}

1. Chambaere K., Roelands M., Deliens L. (2014). Euthanasia for minors in Belgium. JAMA, 312(12), 1258.

2. Grosse C., Grosse A. (2014). Assisted suicide: models of legal regulation in selected European countries and the case law of the European Court of Human Rights. Med Sci Law, pii : 0025802414540636.

3. Mendelson D., Haywood I. (2014). Minors' decision-making capacity to refuse life-saving and life-sustaining treatment: legal and psychiatric perspectives. J Law Med, 21(4), 762-73.

4. Netherlands Ministry of Foreign Affairs. (2010). The termination of life on request and assisted suicide (review procedures) act in practice. Den Haag: Netherlands Ministry of Foreign Affairs.

5. Siegel A.M., Sisti D.A., Caplan A.L. (2014). Pediatric euthanasia in Belgium: disturbing developments. JAMA, 311 (19), 1963-1964.

6. Van Der Heide A. (2013). Assisted suicide and euthanasia. In J.L. Bernat and R. Beresford (Eds), Handbook of Clinical Neurology, Vol. 118 (3rd series) Ethical and Legal Issues in Neurology (pp. 181-89). Elsevier B.V.

\section{Abonnez-vous à \\ Perspectives Psy}

La revue à laquelle vous ne pouvez pas ne pas être abonné

Voir Bulletin d'abonnement page 290 de ce numéro 